\title{
Phenomenological Modeling of DNA Overstretching
}

Ray W. Ogden, Giuseppe Saccomandi, Ivonne Sgura

To cite this article: Ray W. Ogden, Giuseppe Saccomandi, Ivonne Sgura (2011)

Phenomenological Modeling of DNA Overstretching, Journal of Nonlinear Mathematical Physics 18:Supplement 2, 411-427, DOI: https://doi.org/10.1142/S140292511100160X

To link to this article: https://doi.org/10.1142/S140292511100160X

Published online: 04 January 2021 


\title{
PHENOMENOLOGICAL MODELING OF DNA OVERSTRETCHING
}

\author{
RAY W. OGDEN \\ Department of Mathematics, University of Glasgow \\ Glasgow G12 8QW, Scotland, UK \\ and \\ School of Engineering, University of Aberdeen \\ King's College, Aberdeen AB24 3UE, Scotland, UK \\ r.ogden@abdn.ac.uk \\ GIUSEPPE SACCOMANDI \\ Dipartimento di Ingegneria Industriale \\ Università degli Studi di Perugia, 06125 Perugia, Italy \\ saccomandi@mec.dii.unipg.it \\ IVONNE SGURA \\ Dipartimento di Matematica, \\ Università del Salento, 73100 Lecce, Italy \\ ivonne.sgura@unisalento.it
}

Received 23 September 2010 Revised 20 December 2010 Accepted 14 January 2011

\begin{abstract}
A phenomenological model based on the three-dimensional theory of nonlinear elasticity is developed to describe the phenomenon of overstretching in the force-extension curve for doublestranded DNA (dsDNA). By using the concept of a material with multiple reference configurations a single formula is obtained to fit the force-extension curve.
\end{abstract}

Keywords: Force-extension curve; DNA overstretching; nonlinear elasticity; limiting chain extensibility; worm-like-chain formula; nonlinear least squares.

2000 Mathematics Subject Classification: 92C40

\section{Introduction}

A typical force-extension curve for double-stranded DNA (dsDNA) exhibits three portions $[2,17,30]$. During the first portion there is an entropic stretching regime (usually modeled by the worm-like chain), followed by a force plateau in the region of 65 picoNewtons, while in the last portion there is a sharp transition from the usual B-form to a new overstretched form, usually designated S-DNA. The structure of S-DNA remains the subject of debate but it should not be confused with single-stranded DNA (ssDNA) [32, 33]. The biological function of the overstretching DNA transition is complementary to thermal or $\mathrm{pH}$ 
induced denaturation and for this reason there is considerable attention focused on modeling this phenomenon [19], but there appears to be no general agreement about the models that have been proposed in the literature. For example, in a very recent paper [10] it has been shown experimentally that overstretching is more complex than previously considered and that it is in fact a phenomenon that involves two distinct types of double-helix reorganization: slow hysteretic "unpeeling" of one strand off the other; and a fast, non-hysteretic transition to an elongated double-stranded form.

In [24] a model based on a force-induced melting of the DNA double helix was proposed. This implies that S-DNA is made up of a mixture of large islands of separated ssDNA and remnant base-paired B-DNA, and molecular extension is a weighted average of its extension in the two possible states. A two-state worm-like chain (WLC) has been also proposed in [1]. In several papers the idea that the overstretching behavior of DNA may be modeled by a sort of mixture theory has been applied in the study of B-DNA to S-DNA transition as a function of solution conditions, including variations in temperature, $\mathrm{pH}$ and ionic strength (see, for example, [25]). In [3], by using a thermodynamical model for tensionmelted dsDNA it is argued that the overstretching transition cannot be explained in terms of conversion of double helix to noninteracting polynucleotide strands. This is because two parallel noninteracting ssDNAs cannot explain quantitatively the mechanical properties of S-DNA. This is argued directly from an examination of the experimental data by the authors [3]. The Rouzina and Bloomfield model [24, 25] is therefore criticized because in the B-ss scenario the overstretched state should be associated with a constant force between the B-DNA and ssDNA lower than that observed.

The aim of the present note is to present a new framework for describing the overstretching phenomena. Our model is developed using a nonstandard version of the phenomenological theory of nonlinear elasticity where the stress is determined as a function of the deformation gradient calculated with respect to a varying reference configuration in such a way that it is possible to introduce micro-mechanical considerations. This idea was introduced originally by Eckart [9] and then developed more recently by Rajagopal and Wineman $[23,35]$ in order to formulate constitutive equations for materials that undergo deformations induced by microstructural changes. The micro-mechanical interpretation of this theory proposed by De Tommasi et al. in [5], may be quite useful in the study of the overstretching phenomenon.

The general form of the constitutive equation for nonlinear elasticity is expressed in terms of a strain-energy function. In the standard theory it is assumed implicitly that the material response is due to a molecular mechanism that does not change during the deformation process under consideration. Our argument is that in single-molecule experiments on DNA, this assumption may be considered valid only on the first portion of the forceextension curve. At a certain moment the hydrogen bonds between strands start to break and there is a fundamental change in the molecular mechanism responsible for the overall material response. In the case of DNA these microstructural changes are driven by several factors: stretching, salinity, temperature, etc.

The DNA molecule has been modeled mathematically in very different ways. Atomic modeling considers each atom of the molecule as a point mass and interaction laws are introduced via interatomic potentials. Mesoscopic modeling considers base pairs rather than the atoms as the fundamental units and employs semi-empirical laws for the interactions 
between adjacent pairs. Continuum mechanics imagines the DNA to be a continuous filament. Within the continuum framework bending and torsional stiffness are introduced in the usual manner of elasticity theory.

The simple base-pair model provided by Peyrard et al. (see [4] for details) considers that the opposite bases are connected by hydrogen bonds modeled via a Morse potential whereas the bases on the same strand are connected by a simple harmonic potential. Here we adopt a similar micro-mechanical picture. The spring that models the stacking interactions endows the DNA molecule with nonzero stiffness. On the other hand the hydrogen bonds are breakable and are responsible for the alteration of the molecule. We assume that a continuous process of microstructural conversion occurs after the deformation increases beyond a threshold value. In this initial model we shall neglect any factors such as salinity, temperature or ionic strength that are not strictly mechanical. We emphasize that instead of considering the conversion of the double helix to two noninteracting polynucleotide strands, we are considering here a process of conversion related to the rupture of stress-bearing bonds. Upon rupture of the bonds a new microstructural arrangement forms with a new unstressed reference configuration. More details of this constitutive model may be found in $[5]$.

We point out that in the statistical mechanics approach to DNA mechanics it is usual to model this biomolecule as a one-dimensional system. This choice rules out a systematic use of continuum mechanics in DNA mechanics. Sometimes in the framework of these studies researchers tolerate the use of rod theory. On the other hand, experiments such as that reported in [13] show that three-dimensional continuum mechanics may have a fundamental role to play in our understanding of complex deformations of the DNA filament. The reference model from the point of view of statistical mechanics upon which the model of the overstretching phenomenon is based is the discrete persistent chain [29]. Using a technique that has been used to explain the helix-coil transition in biopolymers in [29], the authors develop a seven-parameter model for the overstretching transition with a two-state Ising model living on a discrete persistent chain. We believe that an approach based on continuum mechanics provides a different perspective and may therefore help in understanding this interesting mechanical phenomena.

\section{Basic Equations}

Let us consider a deformation $\mathbf{x}=\mathbf{x}(\mathbf{X}, t)$, where $\mathbf{x}$ is the current position of a particle located at $\mathbf{X}$ in the undeformed configuration at time $t=0$. The deformation gradient is given by $\mathbf{F}(\mathbf{X}, t)=\partial \mathbf{x} / \partial \mathbf{X}$ and the left Cauchy-Green tensor by $\mathbf{B}=\mathbf{F F}^{T}$. We assume that there is a range of deformation for which the material behaves like an incompressible, isotropic elastic material, i.e. the Cauchy stress $\mathbf{T}=-p \mathbf{I}+\mathbf{T}^{(\mathrm{E}, 1)}$, where $-p \mathbf{I}$ is the indeterminate part of the stress due to the constraint of incompressibility ( $\operatorname{det} \mathbf{F}=1$ ) and the extra stress takes the form

$$
\mathbf{T}^{(\mathrm{E}, 1)}=2 W_{1}^{(1)} \mathbf{B}-2 W_{2}^{(1)} \mathbf{B}^{-1} .
$$

The strain-energy function $W^{(1)}=W^{(1)}\left(I_{1}, I_{2}\right)$ is a function of the principal invariants $I_{1}=\operatorname{tr}(\mathbf{B})$ and $I_{2}=\operatorname{tr}\left(\mathbf{B}^{-1}\right)$, where $W_{i}^{(1)}=\partial W^{(1)} / \partial I_{i}, i=1,2$. An activation criterion is needed to determine when the microstructural change begins. This is provided by 
introducing a scalar deformation state parameter $s$. Here, we suppose that $s=s\left(I_{1}, I_{2}\right)$ depends on the deformation through $I_{1}$ and $I_{2}$ for consistency with the requirement of isotropy, although more general forms of $s$ may easily be adopted. For $s<s_{a}$, the threshold value of $s$, no conversion has yet occurred, i.e. all the material is in its original form and the extra stress is given by (2.1). On the other hand, for a value of the state parameter $\widehat{s}$ beyond $s_{a}$ microstructural changes have occurred and the reference configuration has changed. This implies that the stress is now a function of the relative deformation gradient for the material formed at state $\widehat{s}$ given by $\widehat{\mathbf{F}}=\partial \mathbf{x} / \partial \widehat{\mathbf{x}}$, where $\widehat{\mathbf{x}}$ is the position of the particle in the configuration corresponding to deformation state $\widehat{s}$. In Fig. 1 the original reference configuration, the configuration at $\widehat{s}$ and the current configuration are depicted. The associated Cauchy-Green tensor is given by $\widehat{\mathbf{B}}=\widehat{\mathbf{F}} \widehat{\mathbf{F}}^{T}$.

We shall assume that the new material formed at the state $\widehat{s}$ is still elastic, isotropic and incompressible such that the extra Cauchy stress at state $s$ in this new configuration formed at the deformation state $\widehat{s}$ is given by

$$
\mathbf{T}^{(\mathrm{E}, 2)}=2 W_{1}^{(2)} \widehat{\mathbf{B}}-2 W_{2}^{(2)} \widehat{\mathbf{B}}^{-1} .
$$

Here $W^{(2)}=W^{(2)}\left(\widehat{I}_{1}, \widehat{I}_{2}\right)$ is the strain-energy function of the newly formed material, relative to the reference configuration at $\widehat{s}$. Another important simplifying assumption is that a single function $W^{(2)}$ governs the strain energy during the continuous microstructural change. The total current stress is taken as the superposition of the contributions from the material remaining in its original configuration and from all the new material formed at deformation states $\widehat{s} \in\left[s_{a}, s\right]$, i.e.

$$
\mathbf{T}(s)=-p \mathbf{I}+b(s) \mathbf{T}^{(\mathrm{E}, 1)}+\int_{s_{a}}^{s} a(\widehat{s}) \mathbf{T}^{(\mathrm{E}, 2)} d \widehat{s},
$$

where $a(\widehat{s})$ is a conversion function, which measures the "rate" of conversion with respect to the parameter $\widehat{s}$, and $b(s)=1-\int_{s_{a}}^{s} a(\widehat{s}) d \widehat{s}$ is the volume fraction of the material in the original configuration remaining at state $s$.

For $s<s_{a}$, as already indicated, no conversion has yet occurred, all the material is therefore in its original state and the total extra stress is given by (2.1). As the deformation

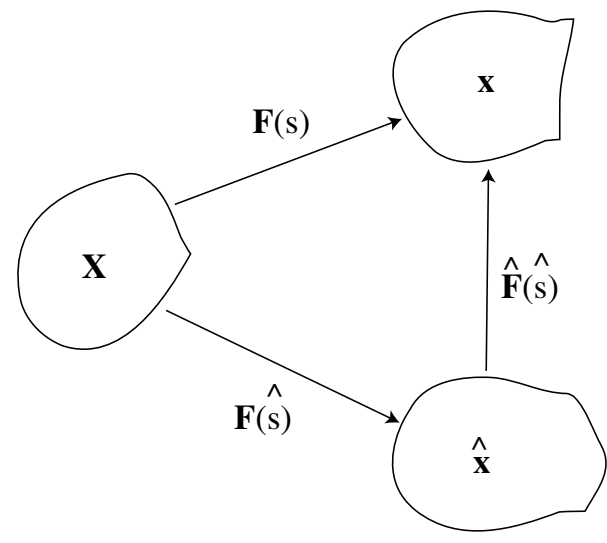

Fig. 1. Schematic illustration of a material with the original reference configuration (left), the evolving reference configuration at the deformation state $\widehat{s}$ and the current configuration. 
proceeds and the state parameter $s$ increases beyond $s_{a}$ the conversion function $a(s)$ determines the amount of material transformed into the new phase.

We require the conversion function to satisfy $a(s)=0$ when $s \leq s_{a}$ and $a(s)>0$ for $s>s_{a}$, while $b(s)=1$ when $s \leq s_{a}$ and $0 \leq b(s)<1$ for $s>s_{a}$. Thus, to complete the model, constitutive equations for $W^{(1)}$ and $W^{(2)}$, the activation criterion $s_{a}$ and the conversion function $a(s)$ have to be prescribed. Our model is three-dimensional and fully consistent with the theory of continuum mechanics. To illustrate the ideas quantitatively we begin with a prototype that is empirical and one-dimensional, and we then show how to recast the theory in three-dimensional form.

\section{The Constitutive Models}

\subsection{Data sources}

We consider a set of experimental data from [32, 33], where several experiments on dsDNA in different salt concentrations were discussed. Here we use measured data $\left(x_{i}, f_{i}\right), i=$ $1, \ldots, m$, corresponding to a force- $(f$ in picoNewtons)-extension ( $x$ in microns $\mu$ ) experiment on a single dsDNA molecule in $250 \mathrm{mM}\left[\mathrm{Na}^{+}\right]$buffer solution at $7.5 \mathrm{pH}$ (these data are reported in Fig. 3 of [32]).

\subsection{An empirical one-dimensional model}

Let $x$ denote the one-dimensional extension. On the same basis as illustrated in Sec. 2, for the one-dimensional force $f$ we have

$$
f(x)=b(x) f^{(1)}(x)+\int_{x_{a}}^{x} a(\widehat{x}) f^{(2)}(\widehat{x}) d \widehat{x} .
$$

In (3.1) the various quantities $a(x), b(x), x_{a}$ have the same meaning as before, with $x$ replacing $s$. Since the process of conversion is continuous we have

$$
b(x)=1-\int_{x_{a}}^{x} a(\widehat{x}) d \widehat{x}, \quad x \geq x_{a} .
$$

The constitutive assumptions we introduce are: for $f^{(1)}$, a logistic modification of the original one-dimensional Fung model widely used in biomechanics [11], i.e.

$$
f^{(1)}(x)=\frac{\mu_{1}}{2} \frac{\exp \left[\beta\left(x-x_{0}\right)\right]}{\exp \left[\beta\left(x-x_{0}\right)\right]+\gamma},
$$

where the material constants $\mu_{1}>0, x_{0}>0$ and $\beta>0$ have dimensions of force, length and 1 /length, respectively, and $\gamma>0$ is a dimensionless constant; and, for $f^{(2)}$, the WLC interpolation formula $[2,14,22]$

$$
f^{(2)}(x)=\mu_{2}\left[\frac{1}{4}(1-z)^{-2}-\frac{1}{4}+z\right] .
$$

Here $\mu_{2}=k T / l_{p}$, where $l_{p}$ is the persistence length, $k$ is Boltzmann's constant and $T$ the absolute temperature (degrees Kelvin), $z=x / l_{c}$ and $l_{c}$ is the contour length of the molecule. Note that, to be consistent with units of the data analysed, the parameters for 
the elastic moduli such as $\mu_{1}, \mu_{2}$ are measured in $\mathrm{pN}$ and for the lengths $\left(l_{c}, x\right.$, etc.) are measured in microns. We have chosen a logistic Fung model to capture the first portion of the force-extension curve (i.e. to capture the strain-hardening phenomenon) but without introducing a singularity such as that in the WLC formula. The WLC is used to model the sharp increase in force at the end of the curve just after the plateau. It is clear that the modeling of the plateau zone depends on how the reference configuration evolves, and this may be controlled by the choice of the conversion function $a(x)$. Usually, in the context of rubber mechanics, very simple models for the conversion function are adopted (for example, quadratic or piecewise linear functional forms). Here we use a functional form suggested by statistical mechanics, namely a probability distribution function computed by considering two possible states for a chain composed of a fixed number of base pairs with a given fixed difference in the energy between the two states. For this purpose, let $c_{1}, c_{2}$ be real constants and

$$
g(x)=\frac{c_{1} e^{-c_{1}\left(x-c_{2}\right)}}{\left[1+e^{-c_{1}\left(x-c_{2}\right)}\right]^{2}} .
$$

Let $\delta$ be a positive constant and define

$$
a(x)=\delta\left(g(x)-g\left(x_{a}\right)\right), \quad x \in\left[x_{a}, x_{c}\right],
$$

with $a(x)=0$ otherwise. Here, we are assuming that the conversion has been completed when $x$ reaches the value $x_{c}$, and this imposes the continuity requirement $a\left(x_{c}\right)=0$, which leads to $c_{2}=\left(x_{a}+x_{c}\right) / 2$. A plot of the function $a(x)$ is shown in Fig. 2 .

We denote by $C$ the total fraction of the material that can undergo conversion. Then,

$$
C=\int_{x_{a}}^{x_{c}} a(\widehat{x}) d \widehat{x}
$$

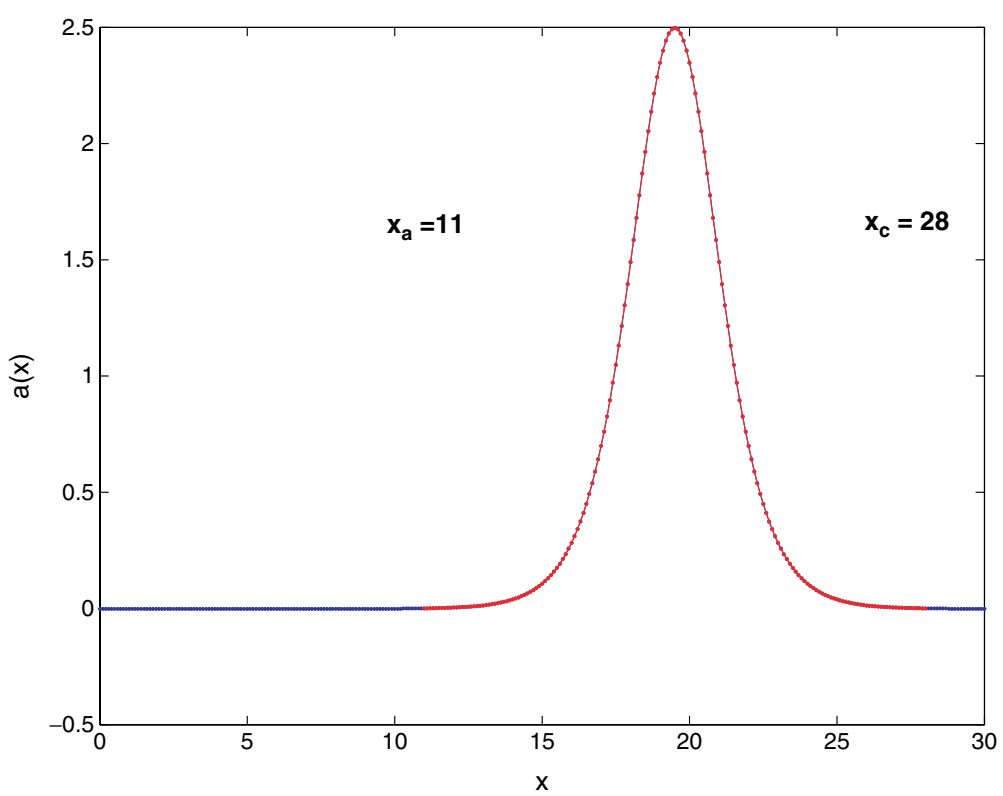

Fig. 2. Plot of the conversion function $a(x)$ against $x$, illustrated for $x_{a}=11$ and $x_{c}=28$ with $c_{1}=1$, based on the set of data in Wenner et al. [32] for the $250 \mathrm{mM}$ buffer solution. 
From the definition of $C$ in (3.6), we calculate the value of $\delta$ by

$$
\delta=\frac{C}{\frac{e^{-c_{1}\left(x_{a}+x_{c}\right) / 2}-e^{-c_{1}\left(x_{c}-x_{a}\right) / 2}}{\left[1+e^{-c_{1}\left(x_{c}-x_{a}\right) / 2}\right]\left[1+e^{-c_{1}\left(x_{a}+x_{c}\right) / 2}\right]}-g\left(x_{a}\right)\left(x_{c}-x_{a}\right)},
$$

and then the constitutive parameters to be identified in this empirical model are $\mu_{1}, \beta, \gamma, \mu_{2}$ and $l_{c}$. Moreover, we have to fix the activation criterion and therefore we also need values for $x_{a}, x_{c}, c_{1}$ and $C$. At this stage the only a priori information about these parameters is that $C \in[0,1]$.

We have to point out two fundamental facts. First, because several parameters have to be identified in the model their numerical approximation could pose severe problems. This is because in the various linear and nonlinear fitting procedures the objective functions may have many local minima, a fact that has been highlighted in [21, 22]. These unpleasant features of least squares problems have also recently been related to the idea of so-called sloppy models. As described in [27] for applications in both biology and physics, this means that the parameters in these multiparameter models can vary in concert by many orders of magnitude without making the fits significantly worse.

The second important issue is to decide whether single molecule experiments are performed in a hard or soft device. This is a commonly used jargon for testing machines in classical experimental mechanics. A hard device is a testing machine where the control parameter is the displacement whereas a soft device is a machine where the control parameter is the load, i.e. the stress (strictly the applied force). When a material with a nonmonotone stressstretch curve is considered, as is usual in the modeling of phase transitions, the difference between the experimental data obtained with the two different protocols is quite important. In the case of nonmonotone curves, roughly speaking, there are two stable branches separated by a metastable region. Each branch corresponds to one of the two coexistent phases. For a soft device, as the load (the control parameter) increases a critical value is reached for which the transition between one phase and the other occurs and it is observed that the displacement suddenly jumps from one stable branch to the other. Such a critical load is computed by means of the Maxwell line. On the other hand, with a hard device where the displacement is controlled it is observed that at the critical load the Maxwell line is traversed from the first stable branch to the second. It seems plausible that in some of the single molecule experiments a hard device is used. The modeling is the same in the case of a soft device but a different approach to the fitting of the experimental data has to be used. From a review of the literature it is clear that the usual micro-manipulation devices are soft devices. In our one-dimensional model this fact is not so important, but in a three-dimensional model the difference is substantial.

For these reasons it is necessary to devise a strategy for the fitting. This strategy is reported in the Appendix and accounts for the physical interpretation of some of these parameters. Among the various parameters it is important to clarify the role of $C$, i.e. of the total fraction of dsDNA converted to sDNA. We know, from [31], for example, and many other studies that not all the dsDNA is converted. In Fig. 3 we show how the conversion is progressing as a function of the elongation $x$. In each nonlinear optimization, the parameters $C, x_{c}, l_{c}$ were fixed, while the others $\left(\mu_{1}, \beta, \gamma, \mu_{2}, c_{1}, x_{a}\right)$ were taken from the fitting procedure on the 1D model. Hence, the curves presented in Fig. 3 correspond to the 


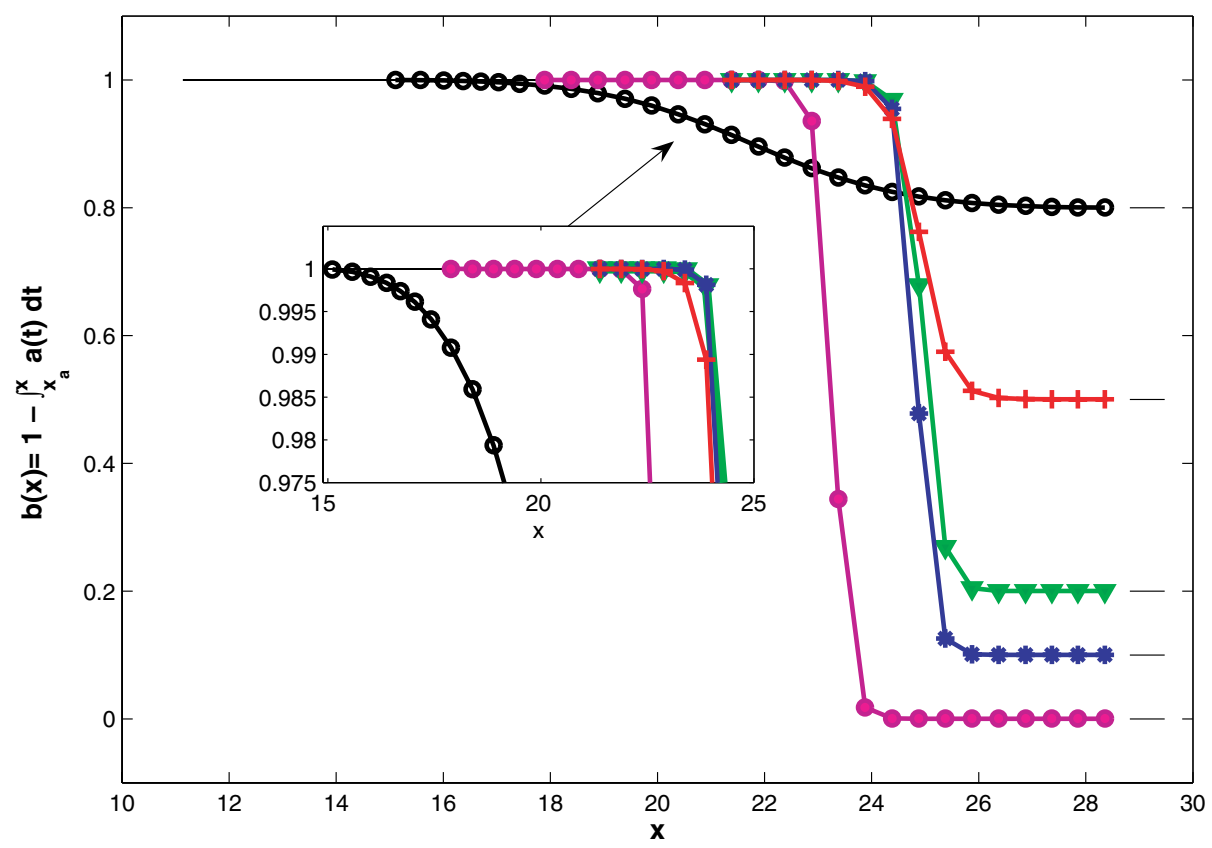

Fig. 3. Plot of the volume fraction $b(x)$ vs $x$ for different values of $C$ in $(3.6): C=0.2, \circ ; C=0.5,+$; $C=0.8, \nabla ; C=0.9, * ; C=1, \bullet$. The conversion is faster and more localized for larger values of $C$. Note that $1-C$ is the fraction of dsDNA remaining at the end of the overstretching transition.

expression (3.2) using the activation function $a(\widehat{x})$ with the parameters $x_{a}$ and $c_{1}$ obtained from the best fit. It is worth noting that for small values of $C$ this conversion process is quite smooth, but beyond a certain level of total conversion becomes very sharp and localized. In our opinion this behavior is reminescent of what happens in thermal denaturation of DNA as reported in the pioneering works by Peyrard et al. (see, for example, [4]). Information of this kind may be used to restrict further the range of variation of the various parameters and it suggests the need for a new set of experiments tailored to the specific model we wish to use. This is exactly what the PNAS commentary [34] points out. At the moment our numerical simulations show that the model proposed here may reproduce experimental data with a fair degree of accuracy. Therefore, the curves presented here are only representative of the peculiarities of the model.

In Fig. 4, we show three predictions of the 1D model obtained for different optimal sets of parameters and with very similar low residuals and errors. These results emphasize the sloppiness of the model [27], or in other words the presence of numerical global minima, i.e. indistinguishable good fits arising from very different parameter arrays.

Remark. We point out that the function $g(x)$ used in the conversion function (3.5) is purely phenomenological, but it is suggested by a simple computation from statistical mechanics. We have supposed that the DNA molecule is composed of a number $N$ of base pairs. A number, say $n_{s}$, of base pairs is in a certain state (for example S-DNA) and the remaining number $n_{b}=N-n_{s}$ is in another state. There is an energy difference $\epsilon$ between the two states. Then a standard computation where thermodynamical equilibrium is assumed based on the minimization of a thermodynamical potential allows $n_{s}$ to be computed as a 
function of $\epsilon$, the applied tensile force and the stretch. We have used this formula to inspire the empirical form of the conversion function.

\subsection{Three-dimensional models}

Now we turn to study the single molecule experiment within the framework of a threedimensional theory. The motivation for this is provided mainly by the findings of [13].
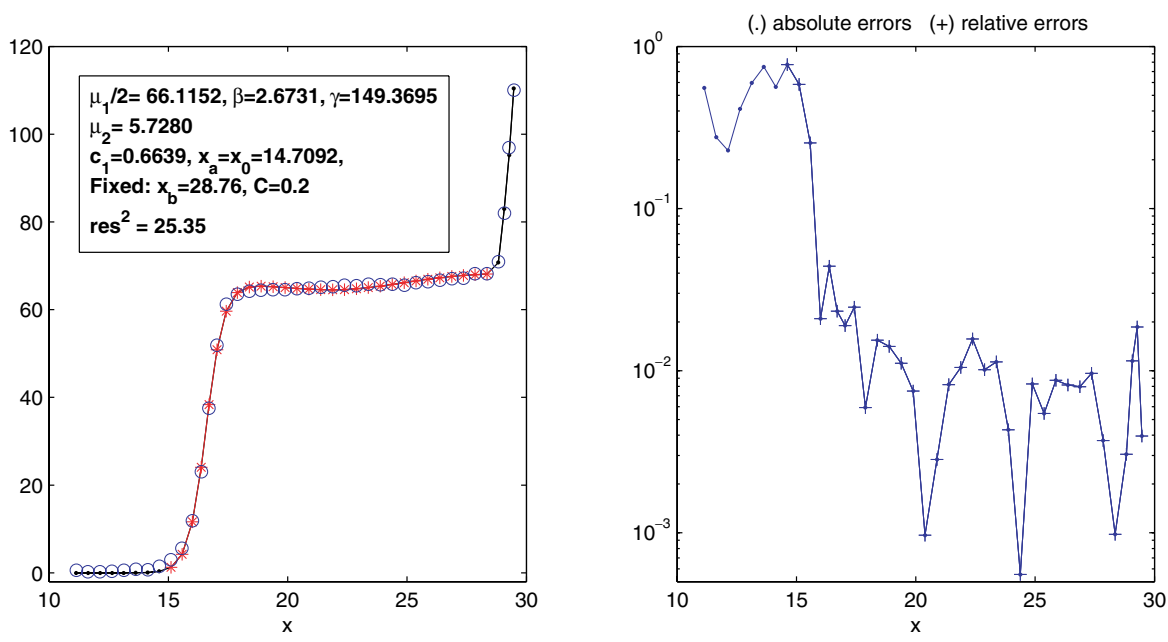

(a)
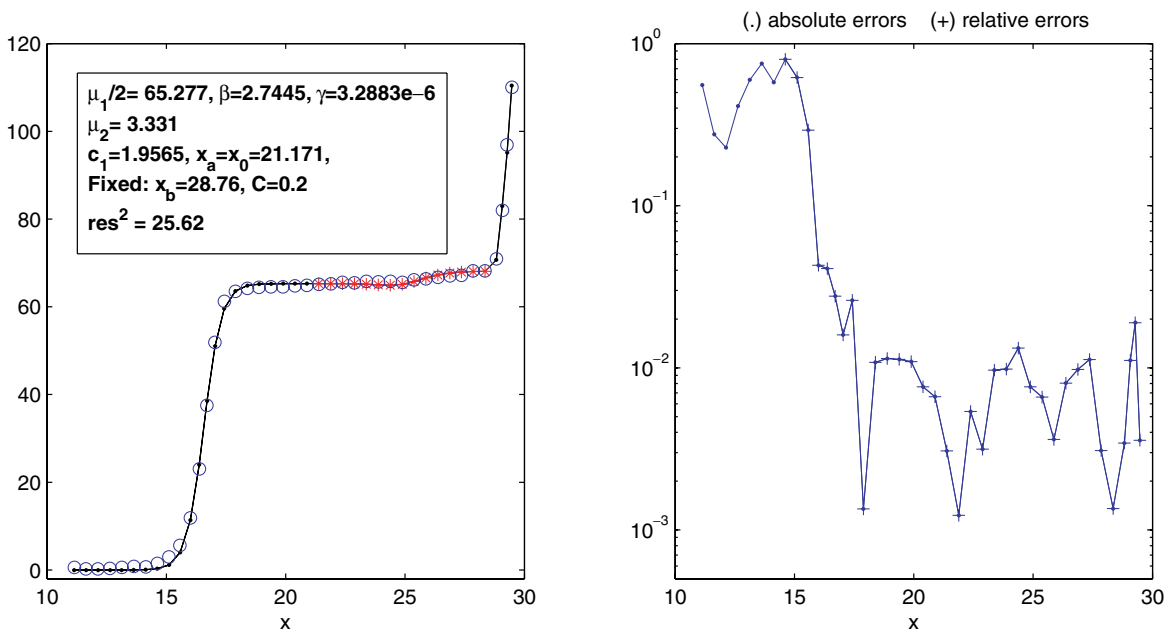

(b)

Fig. 4. Plot of $f(x)$ vs $x$. Circles are from the data in Wenner et al. [32] for the $250 \mathrm{mM}$ buffer solution. In both (a) and (b), the curves in the left panels are obtained from the fitting procedure for the total volume fraction $C=0.2, x_{c}=28.76, l_{c}=32.02$ (contour length). In the left panel of (c) $C=0.8$ instead. The red stars indicate the range of values for which the conversion is active. The corresponding optimal sets of parameter values and the least squares residuals are reported as insets in each plot. In (a), (b) and (c), the right panels show the absolute errors (at the beginning) and relative errors on the data corresponding to the predictions in the corresponding left panels. These results show that we are in the presence of a sloppy model and that quantitative predictions have little meaning. 

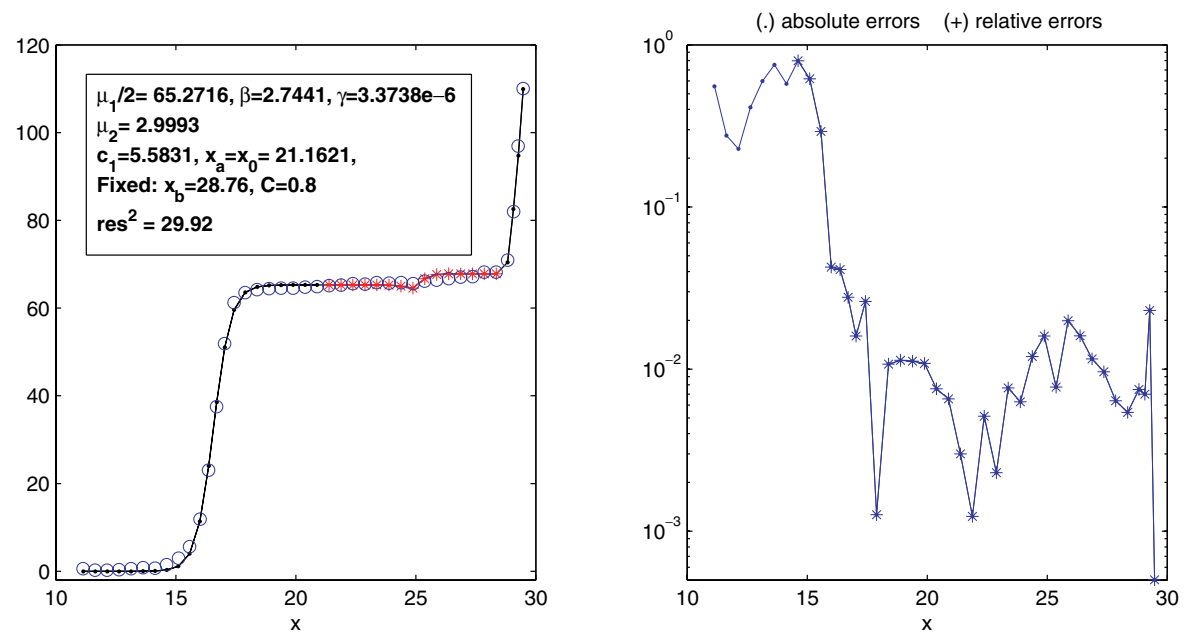

(c)

Fig. 4. (Continued)

In this celebrated paper the authors use a toy model of DNA in which the biomolecule is considered as an incompressible elastic rod wrapped around by a stiff wire. The outer wire is affixed to the inner rod helically and contributes to the overall mechanical properties because it resists stretching and compression. The outer helix increases the torsional rigidity and yields a twist-stretch coupling that depends upon the pitch angle of the helix. Such a twiststretch coupling is well known in the context of three-dimensional continuum mechanics and is referred to as the Poynting effect. For many solids, in particular for rubber-like and polymeric solids, the mechanical response of a tube in torsion is characterized by an elongation related to the square of the amount of twist. This phenomena is clearly and simply explained within the framework of the three-dimensional theory of nonlinear elasticity, but its explanation is much more subtle in the case of one-dimensional theories of rods. Here, because of the available experimental data, we consider just the deformation associated with simple tension, but we show that the ideas of the empirical model introduced in the previous subsection work also in the general setting of continuum mechanics.

We have to point out that the fitting problem for the three-dimensional theory is much harder than for the one-dimensional model of the previous section. Moreover, here it is of central importance to consider if the plateau is a Maxwell line. As will be clear this seems to be the case from several numerical experiments. For all these reasons is not truly significant to compare the fits obtained in the one-dimensional case with that obtained in this section.

In three dimensions the single molecule force-extension experiment is idealized as a simple tension test, for which the deformation is given by

$$
x=\frac{1}{\sqrt{\lambda}} X, \quad y=\frac{1}{\sqrt{\lambda}} Y, \quad z=\lambda Z,
$$

where $\lambda$ is the stretch in the axial (i.e. $z$ ) direction. The current deformation gradient is given in matrix form by $\mathbf{F}(\lambda)=\operatorname{diag}(1 / \sqrt{\lambda}, 1 / \sqrt{\lambda}, \lambda)$, and the corresponding Cauchy-Green deformation matrix is $\mathbf{B}(\lambda)=\operatorname{diag}\left(1 / \lambda, 1 / \lambda, \lambda^{2}\right)$. Hence,

$$
I_{1}=\lambda^{2}+2 \lambda^{-1}, \quad I_{2}=\lambda^{-2}+2 \lambda .
$$


Since this is a one-parameter deformation, it is straightforward to establish that there is a one-to-one correspondence between the activation parameter $s$ and the stretch $\lambda$, and we write $s=s(\lambda)=I_{1}(\lambda)-3$. In fact, it is easy to see that for $\lambda \geq 1$ this is a monotone increasing function and can be inverted. Hence, it is possible to identify an activation stretch, which we denote by $\lambda_{a}$, instead of a generic activation parameter $s_{a}$; then, $s_{a}=s\left(\lambda_{a}\right)=$ $I_{1}\left(\lambda_{a}\right)-3$. The deformation gradient at state $\widehat{\lambda}$ is denoted by $\widehat{\mathbf{F}}(\widehat{\lambda})=\mathbf{F}(\lambda) \mathbf{F}^{-1}(\widehat{\lambda})$ and we compute

$$
\begin{aligned}
& \widehat{\mathbf{F}}(\lambda)=\operatorname{diag}(\sqrt{\widehat{\lambda} / \lambda}, \sqrt{\widehat{\lambda} / \lambda}, \lambda / \widehat{\lambda}), \\
& \widehat{\mathbf{B}}(\lambda)=\operatorname{diag}\left(\widehat{\hat{\lambda}} / \lambda, \widehat{\lambda} / \lambda, \lambda^{2} / \widehat{\lambda}^{2}\right) .
\end{aligned}
$$

It follows that $\widehat{I}_{1}=\lambda^{2} / \widehat{\lambda}^{2}+2 \widehat{\lambda} / \lambda$, and similarly for $\widehat{I}_{2}$.

If we consider the class of elastic materials referred to as generalized neo-Hookean materials, with $W=W\left(I_{1}\right)$, then from (2.1) we obtain the principal components of the Cauchy stress tensor in the form

$$
t_{i}=2 \lambda_{i}^{2} W_{1}-p, \quad i=1,2,3 .
$$

The requirement that the lateral surfaces of the specimen undergoing the simple extension are traction free, $t_{1}=t_{2}=0$, yields

$$
p=2 \lambda^{-1} W_{1}
$$

Generalizing these results to the case (2.3), we see that the tensile force per unit deformed cross-sectional area necessary to achieve the stretch is given by the Cauchy stress component

$$
t_{3}(\lambda)=2 b(\lambda)\left(\lambda^{2}-\lambda^{-1}\right) W_{1}^{(1)}+2 \int_{s_{a}}^{s} a(\widehat{s})\left(\frac{\lambda^{2}}{\lambda(\widehat{s})^{2}}-\frac{\lambda(\widehat{s})}{\lambda}\right) W_{1}^{(2)}(\lambda(\widehat{s})) d \widehat{s},
$$

where

$$
b(\lambda)=1-\int_{s_{a}}^{s} a(\widehat{s}) d \widehat{s}
$$

The corresponding stress per unit undeformed area of cross-section is $F(\lambda)=\lambda^{-1} t_{3}(\lambda)$.

At this point it is necessary to complement (3.12) with the constitutive equations. We need a constitutive equation for the strain-energy function of the material before the conversion starts, i.e. $W^{(1)}$, to model the first portion of the force-extension curve. Then, we also need a constitutive equation for the function $W^{(2)}$ that governs the mechanical behavior of the newly formed material. This choice is important for modeling the "last" portion of the force-extension curve. The overstretching plateau, as already pointed out, is modeled by the choice of the conversion function $a(s)$. For the strain energy $W^{(1)}$ in the first regime we consider a modification of the strain-energy function, here denoted $W^{F}$, based on the original exponential model of Fung for biological tissues given by

$$
W_{1}^{F}=\frac{\mu_{1}}{2} \exp \left[\beta\left(I_{1}-3\right)\right],
$$

where $\mu_{1}>0$ is a constant, here with dimensions of stress, and $\beta>0$ is a dimensionless constant. As for the $1 \mathrm{D}$ case, we need to modify this function because a saturation phenomenon has to be taken into account. The mechanical behavior characterizing the strain 
stiffening of the DNA molecule in the first portion of the force-extension curve cannot influence what happens in the plateau zone. For this reason we consider a logistic modification of the (three-dimensional) Fung model (3.14) analogous to that used for 1D. This is given by

$$
W_{1}^{(1)}=\frac{\mu_{1}}{2} \frac{\exp \left[\beta\left(I_{1}-3\right)\right]}{\exp \left[\beta\left(I_{1}-3\right)\right]+\gamma},
$$

so that

$$
W^{(1)}=\frac{\mu_{1}}{2 \beta} \ln \left(\exp \left(\beta\left(I_{1}-3\right)\right)+\gamma\right),
$$

where $\gamma>0$ is again a dimensionless constant. The function (3.15) reduces to the neoHookean material $W=\mu_{1}\left(I_{1}-3\right) / 2$ when $\gamma=0$.

For the strain-energy function $W^{(2)}$ in the second portion of the deformation range we consider the phenomenological model first proposed by Gent [12] and given by

$$
W^{(2)}\left(\widehat{I}_{1}\right)=-\frac{\mu_{2}}{2} J_{m} \ln \left(1-\frac{\widehat{I_{1}}-3}{J_{m}}\right), \quad \widehat{I}_{1}<J_{m}+3,
$$

where $\mu_{2}$ is the shear modulus for infinitesimal deformations and $J_{m}(>0)$ is the limiting value of $\widehat{I}_{1}-3$ associated with limiting chain extensibility. In the limit as the chain extensibility parameter tends to infinity $\left(J_{m} \rightarrow \infty\right)$, (3.17) also reduces to the classical neoHookean model. The model (3.17) has been discussed in detail by Horgan and Saccomandi [15] and it can be connected with the so-called Freely Jointed Chain (FJC) model. In this case the response function is given by

$$
W_{1}^{(2)}=\frac{\mu_{2}}{2} \frac{J_{m}}{J_{m}-\left(\widehat{I}_{1}-3\right)},
$$

so that the stress has a singularity as $\widehat{I}_{1} \rightarrow J_{m}+3$.

The 3D model we have proposed contains several constitutive parameters that have to be found by using a nonlinear least squares fitting procedure. The parameters needed to fix the strain-energy functions are $\mu_{1}, \beta, \gamma$ and $\mu_{2}, J_{m}$. Moreover, we use the same activation criterion as was used for the $1 \mathrm{D}$ model in (3.5). Hence, to fix the activation criterion we need to identify the interval with ends $x_{a} \rightarrow \lambda_{a}$ and $x_{c} \rightarrow \lambda_{c}$ and the parameters $c_{1}, C$. Note that this criterion may easily be reformulated in a way compatible with $3 \mathrm{D}$ elasticity in terms of the invariant $I_{1}-3$. Equation (3.12) provides a formula for the Cauchy stress, but it is the nominal stress $F(\lambda)=\lambda^{-1} t_{3}(\lambda)$ (force per unit reference cross-sectional area) that is needed for the data fitting. We therefore transform the data set $\left(x_{i}, f_{i}\right)$ into the data set $\left(\lambda_{i}, f_{i}\right)$, where $\lambda_{i}=1+x_{i} / l_{c}$, with the contour length $l_{c}$ identified in the $1 \mathrm{D}$ case. To match the dimensions of the force $f$ in the data the stress $F(\lambda)=\lambda^{-1} t_{3}(\lambda)$ has to be multiplied by the reference cross-sectional area, which is unknown. However, this is just a multiplicative factor that is accounted for by incorporating it into the constants $\mu_{1}$ and $\mu_{2}$, which then have dimensions of force as in the 1D situation.

From the considerations of the 1D model at the end of the previous section and by the arguments in $[31,34]$, a relatively large fraction $C$ of dsDNA can be considered to be converted. Hence, we fix $C$ and try to identify the other parameters such that in the space $(\lambda, F(\lambda))$ the 3D model "sees" the overstretching transition (plateau) in the original data 


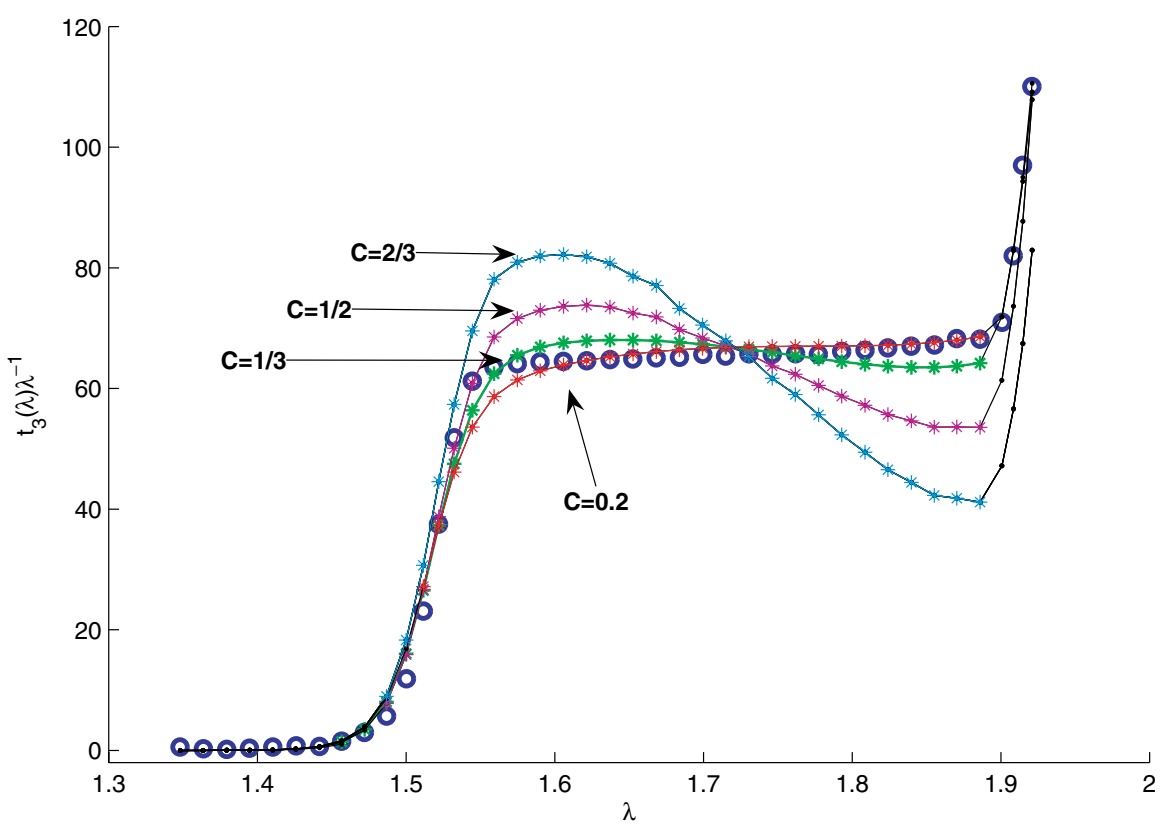

Fig. 5. Plot of $\lambda^{-1} t_{3}(\lambda)$ vs $\lambda$. Circles are from the data in Wenner et al. [32] for the $250 \mathrm{mM}$ buffer solution. The curves are obtained from the fitting procedure, for different (fixed) values of $C$, reported in the plot. The stars indicate the range of values for which the conversion is active. Note that the dimensions of the stress have been converted into "force" by multiplication by the unknown reference cross-sectional area, which is incorporated into the parameters $\mu_{1}$ and $\mu_{2}$.

as its Maxwell line. Curves obtained from the fitting procedure, for different (fixed) values of $C$, are reported in Fig. 5. In this framework, the idea of best fit depends on the kind of device used for the experiments. In a hard device, that is following the data on the plateau, the best fit is obtained for $C=0.2$. In a soft device, it is reasonable to consider as absolute minimizers those having the plateau as their Maxwell line, that is such that the area below and under the plateau are equal. As shown in Fig. 5, this is attained for larger values of $C$.

\section{Concluding Remarks}

The model proposed here yields good fits to the data, and because it has been formulated within a very general framework it may easily be extended to take into account several variables of biological interest, such as solution temperature, $\mathrm{pH}$, and ionic strength (see, e.g., [25]).

It should be mentioned that the number (six) of parameters truly free for fitting in the 1D model is fewer than for some one-dimensional models, such as those studied in [28, 29]. For the 3D model the same number (seven) of free parameters used in the well-known Storm and Nelson model [29] is considered. However, as emphasized in [34], more specific experiments are required to determine all these parameters.

The model is interesting not only because it comprises a single formula describing the complete force-extension curve, but also from a conceptual point of view. Indeed, as has been argued by Cocco et al. [3], S-DNA cannot be described as a simple sort of mixture of dsDNA and ssDNA. The relationship is more complex and it is clarified by the existence 
of multiple reference configurations. We emphasize that because DNA overwinds when it is stretched, in our opinion we need a three-dimensional model to obtain a complete and realistic picture of the single molecule experiments and our model is just a rigorous version of the toy model proposed by Gore et al. in [13].

We have considered that experiments are conducted either with a hard device or a soft device. With a hard device we are able to follow the plateau, i.e. we have an increase of displacement at constant stress, while for a soft device the plateau is the bridge between the two stable branches of the nonmonotone force-extension curve, i.e. we jump from a value of displacement to a significantly larger value.

The mathematical details of the family of models considered here (which were introduced in $[5,6])$ may be found in [7]. The ideas contained in [6] have been applied successfully to the modeling of various biomaterials, including, for example, spider silk [8], and in [16] a similar approach to that in [6] has been used to model beta-sheet proteins.

On the other hand, the need for a mixture theory in modeling DNA mechanics is not new. In 2005 Moakher and Maddocks proposed a theory of birods [20] in which they construct a continuum mechanics model of DNA composed of two elastically interacting elastic strands. The two strands are described in terms of averaged, or macroscopic, variables plus an additional small, internal or microscopic, perturbation. Therefore the model is quite different from that proposed here.

Our results show that not only entropic elasticity, but also the material properties play an important role in DNA mechanics. In the framework of the overstretching transition the role of nonentropic elasticity has now been clearly pointed out by a new generation of experiments [34] that, combining single-molecule force spectroscopy and fluorescence imaging, are able to visualize the phenomena directly. Clearly for our model to be useful more information is needed than is currently available. For example, as already pointed out in the previous section, we need to have clear information about the conversion rates.

When in [31] multi-color, single-molecule fluorescence imaging was used on unconstrained DNA, first the molecule was stretched torsionally to various extensions within the low-force transition and then it was exposed to an intercalating dye. In such a way the experiment was able to reveal the fraction of dsDNA at that extension. This dsDNA fraction corresponds directly to the fraction of the transition remaining to be stretched.

We think that our model may have a deep impact on the nonlinear dynamics of DNA, as already shown in [26]. For example, the constitutive equations considered here introduce the possibility of nonclassical shocks in DNA dynamics, an interesting new idea for understanding DNA denaturation with models à la Peyrard [4]. Indeed, the next step is to consider if, by using a suitable adaptation of the model in [6], we are able to use micromechanical considerations, and not a priori assumptions, to model DNA denaturation based on a nonconvex elastic energy and nonlocal interactions.

\section{Appendix}

The strategy for fitting the theoretical model to the experimental data is based on a nonlinear least squares (NLS) approximation as follows. As a first step, we fix a priori some parameters from simple biological and physical considerations and we solve the optimization problem for the remaining parameters in order to identify a first optimal subset. 
In the successive steps, the strategy consists of implementing the NLS algorithm by starting from this solution and then moving in a descent direction by including each time a new free parameter from amongst those that were fixed. The solution found at each step is then used as an initial guess for solving the next NLS problem in which a further parameter has to be identified. Only $x_{c}, l_{c}, C, x_{0}=x_{a}$ for the $1 \mathrm{D}$ model and $C$ for the 3D model are always fixed. This assumption implies that in the final part of the experimental curve, for $x>x_{c}$ (or $\lambda>\lambda_{c}$ ), the material is all converted to its new form. All the computations are performed in Matlab with the lsqcurvefit routine (see [18]) for solving nonlinear least squares problems. We allow the algorithm to perform a maximum of 1000 iterations and stop with tolerances $(\mathrm{tol}=1 \mathrm{e}-10)$ on the residual and $(\mathrm{tol}=1 \mathrm{e}-6)$ on the iterative steps.

For the 1D model, we considered the starting guesses for the nonlinear problems accounting for the following physical features:

- The total contour length $l_{c}$ is chosen to be slightly larger than the last datum value for the extension since its value locates the asymptote of the WLC;

- $x_{0}=x_{a}$ since $x_{0}$ in the logistic function (3.3) identifies the point where the largest growth occurs, and this corresponds to the meaning of $x_{a}$ in the activation criterion;

- Since $\mu_{1}$ corresponds to the horizontal asymptote of the logistic function, its starting guess is set to almost the force value of the plateau in the data; thus, we set $\mu_{1}=62$.

Three optimal final results with almost equivalent low residuals and errors are reported in the text and in Fig. 4, to emphasize the property of sloppiness for the 1D model.

The same fitting strategy used for fitting the data with the 1D empirical model (3.1) is used for the 3D model (3.12). As starting guess, for the activation criterion we fix $\lambda_{c}=1.895$, for the Gent material we fix $\mu_{1}=\mu_{2}$ equal to almost the force corresponding to the plateau, and the parameter $J_{m}$, accounting for the asymptote location, such that $J_{m}=1.9$ is almost the last numerical value available for the stretch data.

Many local minima have been attained in the fitting procedure, and the best results such that the overstretching plateau can be identified as a Maxwell line are reported in Fig. 5 for different values of the total fraction $C$.

\section{Acknowledgments}

We are grateful to Maria Barbi for suggesting the activation criterion and to Kumbakonam Rajagopal for interesting discussions on generalized continuum mechanics. We wish to thank Mark Williams and Victor Bloomfield for furnishing us with the numerical values of the experimental data in [32].

\section{References}

[1] A. Ashan, J. Rudnick and R. Bruinsma, Elasticity theory of the B-DNA to S-DNA transition, Biophys. J. 74 (1998) 132-137.

[2] C. Bouchiat, M. D. Wang, J.-F. Allemand, T. Strick, S. M. Block and V. Croquette, Estimating the persistence length of a worm-like chain molecule from force-extension measurement, Biophys. J. 76 (1999) 409-413.

[3] S. Cocco, J. Yan, J.-F. Léger, D. Chatenay and J. F. Marko, Overstretching and force-driven strand separation of double-helix DNA, Phys. Rev. E 70 (2004) 011910.

[4] T. Dauxois and M. Peyrard, The Physics of Solitons (Cambridge University Press, 2006). 
[5] D. De Tommasi, G. Puglisi and G. Saccomandi, A micromechanics-based model for the Mullins effect, J. Rheol. 50 (2006) 495-512.

[6] D. De Tommasi, G. Puglisi and G. Saccomandi, Localized versus diffuse damage in amophous materials, Phys. Rev. Lett. 100 (2008) 085502.

[7] D. De Tommasi, S. Marzano, G. Puglisi and G. Saccomandi, Localization and stability in a multi-scale model for damageable amorphous solids, Cont. Mech. Thermodyn. 22 (2010) 42-67.

[8] D. De Tommasi, G. Puglisi and G. Saccomandi, Damage, self-healing and hysterisis in spider silk, Biophys. J. 98 (2010) 1941-1948.

[9] C. Eckart, The thermodynamics of irreversible processes. IV. The theory of elasticity and anelasticity, Phys. Rev. 73 (1948) 373-382.

[10] H. Fu, H. Chen, J. F. Marko and J. Yan, Two distinct overstretched DNA states, Nucleic Acids Res. (2010) 1-7. doi:10.1093/nar/gkq309

[11] Y. C. Fung, Elasticity of soft tissue in simple elongation, Am. J. Physiol. 213 (1967) 1532-1544.

[12] A. N. Gent, A new constitutive relation for rubber, Rubber Chem. Technol. 69 (1996) 59-61.

[13] J. Gore, Z. Bryant, M. Nöllmann, M. U. Le, N. R. Cozzarelli and C. Bustamante, DNA overwinds when stretched, Nature 442 (2006) 836-839.

[14] G. A. Holzapfel and R. W. Ogden, On the bending and stretching elasticity of biopolymer filaments, J. Elasticity, DOI: 10.1007/s10659-010-9277-2, online 19 October 2010, in press.

[15] C. O. Horgan and G. Saccomandi, Finite thermoelasticity with limiting chain extensibility, J. Mech. Phys. Solids 51 (2003) 1127-1146.

[16] S. Keten and M. J. Buehler, Strength limit of entropic elasticity in beta-sheet protein domain, Phys. Rev. E 78 (2008) 061913.

[17] J. F. Marko and E. D. Siggia, Stretching DNA, Macromolecules 28 (1995) 8759-8770.

[18] Matlab, The MathWorks, Optimization Toolbox, Release 6.5.

[19] R. Metzler, T. Ambjörnsson, A. Hanke, Y. Zhang and S. Levene, Single DNA conformations and biological function, J. Comput. Theoret. Nanoscience 4(1) (2007) 1-49.

[20] M. Moakher and J. H. Maddocks, A double-strand elastic rod theory, Arch. Rat. Mech. Anal. 177 (2005) 53-91.

[21] R. W. Ogden, G. Saccomandi, I. Sgura, Fitting hyperelastic models to experimental data, Comput. Mech. 34 (2004) 484-502.

[22] R. W. Ogden, G. Saccomandi and I. Sgura, Computational aspects of worm-like-chain interpolation formulas, Comp. Math. Appl. 53 (2007) 576-586.

[23] K. R. Rajagopal and A. Wineman, A constitutive equation for nonlinear solids which undergo deformation induced microstructural changes, Internat. J. Plasticity 8 (1992) 365-395.

[24] I. Rouzina and V. A. Bloomfield, Force-induced melting of the DNA double helix 1: thermodynamic analysis, Biophys. J. 80 (2001a) 882-893.

[25] I. Rouzina and V. A. Bloomfield, Force-induced melting of the DNA double helix 2: effect of solution conditions, Biophys. J. 80 (2001b) 894-900.

[26] G. Saccomandi and I. Sgura, The relevance of nonlinear stacking interactions in simple models of double-stranded DNA, J. R. Soc. Interface 3 (2006) 655-667.

[27] M. K. Transtrum, B. B. Machta and J. P. Sethna, Why are nonlinear fits to data so challenging? Phys. Rev. Lett. 104 (2010) 060201.

[28] A. Sarkar, J.-F. Léger, D. Chatenay and J. F. Marko, Structural transitions in DNA driven by external force and torque, Phys. Rev. E 63 (2001) 051903.

[29] C. Storm and P. C. Nelson, Theory of high-force DNA stretching and overstretching, Phys. Rev. E 67 (2003) 051906.

[30] T. R. Strick, M.-N. Dessinges, G. Charvin, N. H. Dekker, J.-F. Allemand, D. Bensimon and V. Croquette, Stretching of macromolecules and proteins, Rep. Prog. Phys. 66 (2003) 1-45.

[31] J. van Mameren, P. Gross, G. Farge, P. Hooijman, M. Modesti, M. Falkenberg, G. J. L. Wuitea and E. J. G. Peterman, Unraveling the structure of DNA during overstretching by using multicolor, single-molecule fluorescence imaging, PNAS 106 (2009) 18231-18236. 
[32] J. R. Wenner, M. C. Williams, I. Rouzina and V. A. Bloomfield, Salt dependence of the elasticity and overstretching transition of single DNA molecules, Biophys. J. 82 (2002) 3160-3169.

[33] M. C. Williams, I. Rouzina and V. A. Bloomfield, Thermodynamical of DNA interactions from single molecule stretching experiments, Acc. Chem. Res. 35 (2002) 159-166.

[34] M. C. Williams, I. Rouzina and M. J. McCauley, Peeling back the mystery of DNA overstretching, PNAS 106 (2009) 18047-18048.

[35] A. Wineman and K. R. Rajagopal, On a constitutive theory for materials undergoing microstructural changes, Arch. Mech. 42 (1990) 53-74. 